Int. Journal of Math. Analysis, Vol. 7, 2013, no. 2, 73 - 83

\title{
Strong and Semi Strong Outer Mod Sum Graphs
}

\author{
M. Jayalakshmi and B. Sooryanarayana \\ Dept.of Mathematical and Computational Studies \\ Dr.Ambedkar Institute of Technology, Bangalore \\ Karnataka State, India, Pin 560056 \\ jayachatra@yahoo.co.in, dr_bsnrao@yahoo.co.in
}

\begin{abstract}
A semi strong outer mod sum labeling of a graph $G$ is an injective mapping $f: V(G) \rightarrow Z^{+}$with an additional property that for each vertex $v$ of $G$, there exist vertices $w_{1}, w_{2}$ in $V(G)$ such that $f\left(w_{1}\right)=\sum_{u \in N(v)} f(u)$ and $f(v)=\sum_{u \in N\left(w_{2}\right)} f(u)$, where both the sums are taken under addition modulo $m$ for some positive integer $m$. A graph $G$ which admits a semi strong outer mod sum labeling is called a semi strong outer mod sum graph. In this paper we show that the Paths $P_{n}$ for all $n \neq 3$, the Cycle $C_{6}$, and the Complete graphs $K_{n}$ for all $n \neq 3$ are semi strong outer mod sum graphs.
\end{abstract}

Mathematics Subject Classification: 05C78, 05C12, 05C15

Keywords: Sum labeling, Mod Sum labeling, Outer Sum labeling, Outer mod Sum labeling

\section{Introduction}

All the graphs considered here are non trivial, undirected, finite, connected and simple. We use the standard terminology, the terms not defined here may be found in [2] and [3]

A sum labeling $\lambda$ of a graph is a mapping of the vertices of $G$ into distinct positive integers such that for $u, v \in V(G), u v \in E(G)$ if and only if $\lambda(u)+$ $\lambda(v)=\lambda(w)$ for some vertex $w$ of $G$. A graph which admits a sum labeling is called a sum graph. Sum graph were originally proposed by F. Harary [8] and later he extended to include all integers in [9]. Similarly, Bolan, Laskar, Turner and Domke [1] introduced a mod sum labeling of a graph in which the sum $\lambda(u)+\lambda(v)$ is taken under addition modulo $m$ for some positive integer $m$.

An outer sum labeling $f$ of a non-trivial graph is a mapping of the vertices of $G$ into distinct positive integers such that for each vertex $v \in V(G)$, there exists 
a vertex $w \in V(G)$ with $f(w)=\sum_{u \in N(v)} f(u)$, where $N(v)=\{x: v x \in E(G)\}$. A graph $G$ which admits an outer sum labeling is called an outer sum graph. If $G$ is not an outer sum graph, then by adding certain number of isolated vertices to $G$, we can make the resultant graph an outer sum graph. The minimum of such isolated vertices required for a graph $G$, to make the resultant graph an outer sum graph, is called the outer sum number of $G$ and is denoted by on $(G)$. Outer sum graph were originally proposed by B. Sooryanarayana, Manjula K and Vishu Kumar M [14]. Further Jayalakshmi M, B. Sooryanarayana, P Devadas Rao [10] introduced a outer mod sum labeling of a graph. An outer mod sum labeling of a graph $G$ is an injective mapping $f: V(G) \rightarrow Z^{+}$with an additional property that for each vertex $v$ of $G$, there is a vertex $w$ in $G$ such that $f(w)=\sum_{u \in N(v)} f(u)$, where the sum is taken under addition modulo $m$ for some fixed positive integer $m$. The vertex $w$ is then called a working vertex for $v$ and in particular if $\sum_{u \in N(v)} f(u)=f(v)$, then vertex $v$ is called a self working vertex. A graph $G$ which admits an outer mod sum labeling is called an outer mod sum graph (OMSG). For the entire survey on sum labeling and mod sum labeling we refer the latest survey article by Joseph A. Gallian [6].

The purpose of this paper is to introduce the special types of outer mod sum graphs and their properties.

\section{Strong and Semi Strong Outer Mod Sum Graphs}

A semi-strong outer mod sum labeling (SSOMSL) $f$ of a graph $G$ is an outer mod sum labeling of $G$ with an additional property that every vertex of $G$ is a working vertex of some vertex of $G$. Further a semi strong outer mod sum labeling $f$ of a graph $G$, under which every vertex of $G$ is a self working vertex is called a strong outer mod sum labeling (SOMSL). A graph $G$ which admits a semi-strong outer mod sum labeling is called a semi-strong outer mod sum graph (SSOMSG). Similarly, a strong outer mod sum graph (SOMSG) is defined. For notational convenience, the sum, $\sum_{u \in N(v)} f(u)$ is denoted by $N_{f}(v)$ and call it as $f$-neighborhood sum of the vertex $v$ and that of under modulo $m$ is denoted by $N_{f_{m}}(v)$ and call it as $f$-neighborhood sum of the vertex $v$ under modulo $m$.

Example 1 Consider the labeling $f: V\left(P_{5}\right) \rightarrow Z^{+}$defined by $f\left(v_{1}\right)=4$, $f\left(v_{2}\right)=7, f\left(v_{3}\right)=5, f\left(v_{4}\right)=8, f\left(v_{5}\right)=9$, as shown in the figure 1 .

Under the addition modulo 10, we see that the working vertex of $v_{1}$ is $v_{2}$ and $v_{1}$ is the working vertex of $v_{4}$; the working vertex of $v_{2}$ is $v_{5}$ and $v_{2}$ is the working vertex of $v_{1} ; v_{3}$ is the self working vertex; the working vertex of $v_{4}$ is $v_{1}$ and $v_{4}$ is the working vertex of $v_{5}$; the working vertex of $v_{5}$ is $v_{4}$ and $v_{5}$ is 


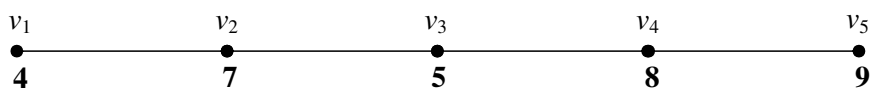

Figure 1: A SSOMSL of the graph $P_{5}$.

the working vertex of $v_{2}$. So $P_{5}$ is a semi-strong outer mod sum graph.

Example 2 Consider the labeling $f: V\left(C_{6}\right) \rightarrow Z^{+}$defined by $f\left(v_{1}\right)=1$, $f\left(v_{2}\right)=3, f\left(v_{3}\right)=2, f\left(v_{4}\right)=9, f\left(v_{5}\right)=7, f\left(v_{6}\right)=8$, as shown in the figure 2.

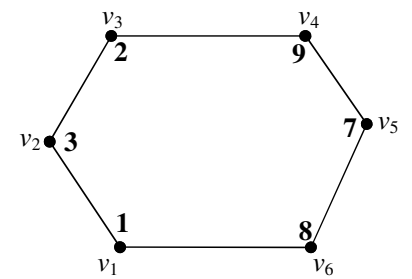

Figure 2: A SOMSL of the graph $C_{6}$.

Under addition modulo 10, $N_{f_{10}}\left(v_{1}\right)=1=f\left(v_{1}\right), N_{f_{10}}\left(v_{2}\right)=3=f\left(v_{2}\right)$, $N_{f_{10}}\left(v_{3}\right)=2=f\left(v_{3}\right), N_{f_{10}}\left(v_{4}\right)=9=f\left(v_{4}\right), N_{f_{10}}\left(v_{5}\right)=7=f\left(v_{5}\right), N_{f_{10}}\left(v_{6}\right)=$ $8=f\left(v_{6}\right)$. Therefore, $v_{i}$ is a self working vertex for each $i, 1 \leq i \leq 6$ and hence $C_{6}$ is a strong outer mod sum graph.

By the definition it follows that every SOMSL is SSOMSL and every SSOMSL is OMSL. Thus we conclude;

Lemma 2.1. Every strong outer mod sum graph is a semi strong outer mod sum graph.

The converse of the lemma 2.1 need not be true in general. As a counter example we see that from example 1 , the path $P_{5}$ is semi strong outer mod sum graph. But path $P_{5}$ is not strong outer mod sum graph. Because, the working vertex of $v_{1} \in P_{5}$ is always $v_{2}$ with respect to any labeling $f$.

Lemma 2.2. Every semi strong outer mod sum graph is an outer mod sum graph.

The converse of the lemma 2.2 need not be true in general. As a counter example the path $P_{3}$ is an outer mod sum graph. But the path $P_{3}$ is not semi strong outer mod sum graph. Because

In figure $3, v_{2}$ is the working vertex of both $v_{1}$ and $v_{3}$. Therefore at the most either $v_{1}$ or $v_{3}$ will be the working vertex of $v_{2}$. So that the vertex $v_{1}$ or $v_{3}$ will not be the working vertex of any of the vertices of $P_{3}$, under any labeling $f$ and any addition modulo $m$. Hence $P_{3}$ is not a semi strong outer mod sum graph. 


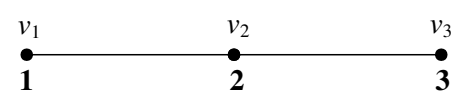

Figure 3: An example for a graph which is not a SSOMSG.

Lemma 2.3. A graph $G$ is a semi strong outer mod sum graph if and only if each vertex of $G$

(i) has a unique working vertex in $G$ and

(ii) is the working vertex of exactly one of the vertex of $G$.

Proof. Let $G$ be a semi-strong outer mod sum graph with $n$ vertices $v_{1}, v_{2}, v_{3}$, $\ldots, v_{n}$. Then, by the definition of SSOMSG, there exists a SSOMSL $f$ under which ,

i) each vertex $v_{i}$ of $G$ has a working vertex in $G$ and is unique (since $f$ is injective), and

ii) each vertex of $G$ is a working vertex of some vertex of $G$. Let $w_{i} \in V(G)$ be the working vertex of $v_{i}$ for each $i=1,2, \ldots, n$. We now see that $w_{i}^{\prime} s$ are all distinct. In fact, if $w_{i}=w_{j}$ for some $i \neq j$ then, as $f$ is injective, at least one vertex in $V(G)$ is not a working vertex of any vertex of $G$, which is a contradiction.

Converse part follows directly by the definition.

Remark 2.4. The Lemma 2.3 says that a graph $G$ is a semi-strong outer mod sum graph if and only if $N_{f_{m}}\left(v_{i}\right) \neq N_{f_{m}}\left(v_{j}\right)$ for all $i \neq j$.

By Remark 2.4, we immediately conclude the following;

Theorem 2.5. The cycle $C_{4}$ is not a SSOMSG and hence not a SOMSG.

Theorem 2.6. Let $G$ be a r-regular graph and $f$ be a SSOMSL of $G$, under modulo $m$. Then $(r-1) \sum_{v_{i} \in V(G)} f\left(v_{i}\right) \equiv 0(\bmod m)$ and hence $\sum_{v_{i} \in V(G)} f\left(v_{i}\right) \equiv$ $0(\bmod m)$ if $\operatorname{gcd}(r-1, m)=1$.

Proof. Let $G$ be a $r$ - regular graph having the vertices $v_{1}, v_{2}, v_{3}, \ldots, v_{n}$ and let $f$ be any SSOMSL of $G$ under modulo $m$. Then each vertex $v_{i}$ of $G$ is the working vertex of exactly one of the vertices of $G$. Also $\operatorname{deg} v_{i}=r$ which means that each vertex $v_{i}$ has $r$ neighborhoods and hence $f\left(v_{i}\right)$ contribute for $f$-neighborhood sum of exactly $r$ vertices. Therefore, $\sum_{v_{i} \in V(G)} f\left(v_{i}\right)=$ $\sum_{v_{j} \in V(G)} N_{f_{m}}\left(v_{j}\right) \equiv\left[\sum_{v_{i} \in V(G)} r \times f\left(v_{i}\right)\right](\bmod m) \Rightarrow(r-1) \sum_{v_{i} \in V(G)} f\left(v_{i}\right) \equiv$ $0(\bmod m) \Rightarrow \sum_{v_{i} \in V(G)} f\left(v_{i}\right) \equiv 0(\bmod m)$ if $(r-1, m)=1$. 
Since the cycle $C_{n},(n \geq 3)$ is a 2-regular graph, it follows immediately, as a consequence of the above Theorem 2.6, that:

Corollary 2.7. If $f$ is a SSOMSL of a Cycle $C_{n}(n \geq 3)$ under modulo $m$, then

$$
\sum_{v_{i} \in V(G)} f\left(v_{i}\right) \equiv 0(\bmod m)
$$

\section{SSOMSL of a Path}

Throughout this section, $v_{1}, v_{2}, \ldots, v_{n}$ denote the vertices of the path $P_{n}$ such that $v_{i}$ is adjacent to $v_{j}$ if and only if $|i-j|=1$ for each $i, j, 1 \leq i, j \leq n$.

Theorem 3.1. For $n=2$ or any integer $n \geq 4$, the path $P_{n}$ is a SSOMSG under modulo $m(>n)$.

Proof. For $n=2,4,6$, it is easy to verify that the function $f: V(G) \rightarrow Z^{+}$ defined by $f\left(v_{i}\right)=i$ serves as a SSOMSL under modulo $n+1$ and the case $n=5$ follows by the Figure 1 under modulo 10 .

For $n \geq 7$ and any $l \in Z^{+}$, define $f: V\left(P_{n}\right) \rightarrow Z^{+}$as follows.

$$
f\left(v_{i}\right)= \begin{cases}l, & \text { if } i=1 \\ l+1, & \text { if } i=3 \\ f\left(v_{1}\right)+f\left(v_{3}\right), & \text { if } i=4 \\ f\left(v_{4}\right)+1, & \text { if } i=2 \\ f\left(v_{k-1}\right)+f\left(v_{k-3}\right), & \text { if } i=k \text { for any } k \in Z^{+} \text {with } 4<k<n-1 \\ f\left(v_{n-2}\right)+f\left(v_{n-4}\right), & \text { if } i=n \\ m+f\left(v_{3}\right)-f\left(v_{n-3}\right), & \text { if } i=n-1 \text { where } m=f\left(v_{n}\right)+f\left(v_{n-2}\right)-f\left(v_{1}\right)\end{cases}
$$

The function $f$ defined above is clearly injective. We now show that it is a SSOMSL of $P_{n}$. In fact,

i) $N_{f}\left(v_{1}\right)=f\left(v_{2}\right)$ and $N_{f}\left(v_{n-1}\right)=f\left(v_{n}\right)+f\left(v_{n-2}\right)=m+f\left(v_{1}\right) \equiv f\left(v_{1}\right)(\bmod$ $m) \Rightarrow$ the working vertex of $v_{1}$ is $v_{2}$ and $v_{1}$ is the working vertex of $v_{n-1}$.

ii) $N_{f}\left(v_{2}\right)=f\left(v_{1}\right)+f\left(v_{3}\right)=f\left(v_{4}\right)$ and $N_{f}\left(v_{1}\right)=f\left(v_{2}\right) \Rightarrow$ the working vertex of $v_{2}$ is $v_{4}$ and $v_{2}$ is the working vertex of $v_{1}$.

iii) $N_{f}\left(v_{3}\right)=f\left(v_{2}\right)+f\left(v_{4}\right)=f\left(v_{5}\right)$ and $N_{f}\left(v_{n-2}\right)=f\left(v_{n-1}\right)+f\left(v_{n-3}\right)=$ $m+f\left(v_{3}\right) \equiv f\left(v_{3}\right)(\bmod m) \Rightarrow$ the working vertex of $v_{3}$ is $v_{5}$ and $v_{3}$ is the working vertex of $v_{n-2}$.

iv) For each $k \in Z^{+}$with $4 \leq k \leq n-4, N_{f}\left(v_{k}\right)=f\left(v_{k-1}\right)+f\left(v_{k+1}\right)=$ $f\left(v_{k+2}\right)$ and $N_{f}\left(v_{k-2}\right)=f\left(v_{k-1}\right)+f\left(v_{k-3}\right)=f\left(v_{k}\right) \Rightarrow$ the working vertex of $v_{k}$ is $v_{k+2}$ and $v_{k}$ is the working vertex of $v_{k-2}$. 
v) $N_{f}\left(v_{n-3}\right)=f\left(v_{n-2}\right)+f\left(v_{n-4}\right)=f\left(v_{n}\right)$ and $N_{f}\left(v_{n-5}\right)=f\left(v_{n-6}\right)+$ $f\left(v_{n-4}\right)=f\left(v_{n-3}\right) \Rightarrow$ the working vertex of $v_{n-3}$ is $v_{n}$ and $v_{n-3}$ is the working vertex of $v_{n-5}$.

vi) $N_{f}\left(v_{n-2}\right)=f\left(v_{n-1}\right)+f\left(v_{n-3}\right)=m+f\left(v_{3}\right) \equiv f\left(v_{3}\right)(\bmod m)$ and $N_{f}\left(v_{n-4}\right)=$ $f\left(v_{n-5}\right)+f\left(v_{n-3}\right)=f\left(v_{n-2}\right) \Rightarrow$ the working vertex of $v_{n-2}$ is $v_{3}$ and $v_{n-2}$ is the working vertex of $v_{n-4}$.

vii) $N_{f}\left(v_{n-1}\right)=f\left(v_{n-2}\right)+f\left(v_{n}\right)=m+f\left(v_{1}\right) \equiv f\left(v_{1}\right)(\bmod m)$ and $N_{f}\left(v_{n}\right)=$ $f\left(v_{n-1}\right) \Rightarrow$ the working vertex of $v_{n-1}$ is $v_{1}$ and $v_{n-1}$ is the working vertex of $v_{n}$.

viii) $N_{f}\left(v_{n}\right)=f\left(v_{n-1}\right)$ and $N_{f}\left(v_{n-3}\right)=f\left(v_{n-2}\right)+f\left(v_{n-4}\right)=f\left(v_{n}\right) \Rightarrow$ the working vertex of $v_{n}$ is $v_{n-1}$ and $v_{n}$ is the working vertex of $v_{n-3}$.

Hence the result.

Open Problem 3.2. Certain values of $m$ considered in the above theorem are not optimal. It is exponentially increasing with $n$ as shown in the table 1. The next Theorem 3.3 gives an optimal value for $m$, whenever $n$ is even. Determine the optimal value of $m$ for the case $n$ is odd.

\begin{tabular}{|c|c|}
\hline Value of $n$ & Value of $m$ \\
\hline 7 & 15 \\
\hline 8 & 20 \\
\hline 9 & 30 \\
\hline 10 & 46 \\
\hline 11 & 67 \\
\hline 12 & 98 \\
\hline 13 & 145 \\
\hline 14 & 213 \\
\hline 15 & 312 \\
\hline 16 & 458 \\
\hline 17 & 672 \\
\hline 18 & 985 \\
\hline 19 & 1444 \\
\hline
\end{tabular}

\begin{tabular}{|c|c|}
\hline Value of $n$ & Value of $m$ \\
\hline 20 & 2117 \\
\hline 21 & 3103 \\
\hline 22 & 4548 \\
\hline 23 & 6666 \\
\hline 24 & 9770 \\
\hline 25 & 14319 \\
\hline 26 & 20986 \\
\hline 27 & 30757 \\
\hline 28 & 45077 \\
\hline 29 & 66064 \\
\hline 30 & 96822 \\
\hline 31 & 141900 \\
\hline 32 & 207965 \\
\hline
\end{tabular}

Table 1: The values of $m$ and $n$ (when $l=1$ ) for a SSOMSL of the graph $P_{n}$ as in the proof of Theorem 3.1.

Theorem 3.3. For any integer $k \geq 1$, the path $P_{2 k}$ is a SSOMSG under modulo $2 k+1$. 


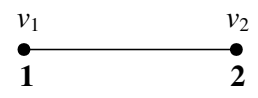

Figure 4: An SSOMS-labeling of $P_{2}$.

Proof. Let $n=2 k$. Now, the proof when $k=1$ follows from the Figure 4 as $v_{2}$ is the working vertex of $v_{1}$ and vice versa.

For $k \geq 2$, define $f: V\left(P_{n}\right): \rightarrow\{1,2, \ldots, n\}$ by $f\left(v_{i}\right)=i$ for all $i, 1 \leq i \leq n$. Then $N_{f}\left(v_{1}\right)=f\left(v_{2}\right)=2$ and $N_{f}\left(v_{n}\right)=f\left(v_{n-1}\right)=n-1$, implies that the

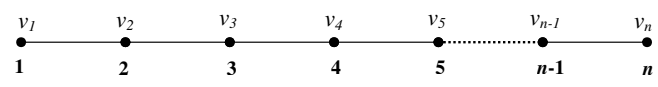

Figure 5: An SSOMS-labeling of even path.

working vertex of $v_{1}$ is $v_{2}$ and the working vertex of $v_{n}$ is $v_{n-1}$. Also for all $j \in Z^{+}, 2 \leq j \leq n-1, N_{f}\left(v_{j}\right)=f\left(v_{j-1}\right)+f\left(v_{j+1}\right)=j-1+j+1=2 j$. Therefore, if $2 \leq j \leq \frac{n}{2}$, then $4 \leq N_{f_{n+1}}\left(v_{j}\right)=2 j=f\left(v_{2 j}\right) \leq n$, which implies that the working vertex of $v_{2}, v_{3}, \ldots, v_{\frac{n}{2}}$ are respectively $v_{4}, v_{6}, \ldots, v_{n}$ and if $\frac{n}{2}+1 \leq j \leq n-1$, then $1 \leq N_{f_{n+1}}\left(v_{j}\right)=2 j-n-1=f\left(v_{2 j-n-1}\right) \leq n-3$, which implies that the working vertex of $v_{\frac{n}{2}+1}, v_{\frac{n}{2}+2}, \ldots, v_{n-1}$ are respectively $v_{1}, v_{3}$, $\ldots, v_{n-3}$. Thus each vertex has a unique working vertex and each vertex is the working vertex of exactly one of the vertex. So $f$ is a SSOMSL and hence the theorem.

Remark 3.4. The labeling $f: V\left(P_{n}\right): \rightarrow\{1,2, \ldots, n\}$, defined by $f\left(v_{k}\right)=k$ as in the theorem 3.3, is not a semi strong outer mod sum labeling under addition modulo $n+1$ if $n$ is odd (since $v_{k}$ and $v_{k+\frac{n+1}{2}}$ have the common working vertex).

In every path $P_{n}$ and any injective labeling $f: V(G) \rightarrow Z^{+}$, working vertex of $v_{1}$ is always $v_{2}$ and working vertex of $v_{n}$ is always $v_{n-1}$. So, $v_{1}$ and $v_{n}$ can not be the self working vertices under any modulo $m$. Thus;

Theorem 3.5. For $n=2$ and any integer $n \geq 4$, the path $P_{n}$ is a SSOMSG and is not a SOMSG.

\section{SOMSL and SSOMSL of a Cycle}

Let $v_{1}, v_{2}, \ldots, v_{n}$ be the vertices of the cycle $C_{n}$ with $v_{i}$ adjacent to $v_{i+1}$ for all $i, 1 \leq i \leq n-1$ and $v_{n}$ adjacent to $v_{1}$.

Theorem 4.1. For any integer $n \geq 3$, the Cycle $C_{n}$ is a SOMSG if and only if $n=6$. 
Proof. In view of Figure 2, it suffices to show that the cycle $C_{n}$ is not a SOMSG whenever $n \neq 6$. We first consider the case $n \geq 7$. If possible suppose that $f: V\left(C_{n}\right) \rightarrow Z^{+}$be an injective function such that $f\left(v_{i}\right)=N_{f_{m}}\left(v_{i}\right)$ for some $m \in Z^{+}$with $m>n$ and for all $i, 1 \leq i \leq n$. Without loss of generality we assume that $f\left(v_{1}\right)=l<f\left(v_{3}\right)=k$, Clearly $l, k<m$

Case 1: $l+k<m$

In this case,

- $N_{f}\left(v_{2}\right)=f\left(v_{1}\right)+f\left(v_{3}\right)=l+k \Rightarrow f\left(v_{2}\right)=N_{f_{m}}\left(v_{2}\right)=l+k(\because$ $l+k<m)$.

- $N_{f}\left(v_{3}\right)=f\left(v_{2}\right)+f\left(v_{4}\right)=l+k+f\left(v_{4}\right) \Rightarrow k=f\left(v_{3}\right)=N_{f_{m}}\left(v_{3}\right) \equiv$ $l+k+f\left(v_{4}\right)(\bmod m) \Rightarrow m=l+f\left(v_{4}\right) \Rightarrow f\left(v_{4}\right)=m-l$.

- $N_{f}\left(v_{4}\right)=f\left(v_{3}\right)+f\left(v_{5}\right)=k+f\left(v_{5}\right) \Rightarrow f\left(v_{4}\right)=N_{f_{m}}\left(v_{4}\right) \Rightarrow m-l \equiv$ $k+f\left(v_{5}\right)(\bmod m) \Rightarrow f\left(v_{5}\right)=m-l-k(\because m>m-(l+k)>0)$.

- $N_{f}\left(v_{5}\right)=f\left(v_{4}\right)+f\left(v_{6}\right)=m-l+f\left(v_{6}\right) \Rightarrow f\left(v_{5}\right)=N_{f_{m}}\left(v_{5}\right) \Rightarrow$ $m-l-k \equiv m-l+f\left(v_{6}\right)(\bmod m) \Rightarrow f\left(v_{6}\right)=m-k$.

- $N_{f}\left(v_{6}\right)=f\left(v_{5}\right)+f\left(v_{7}\right)=m-l-k+f\left(v_{7}\right) \Rightarrow m-k=f\left(v_{6}\right)=$ $N_{f_{m}}\left(v_{6}\right) \equiv m-l-k+f\left(v_{7}\right)(\bmod m) \Rightarrow f\left(v_{7}\right)=l$.

Case 2: $l+k>m$

- $N_{f}\left(v_{2}\right)=f\left(v_{1}\right)+f\left(v_{3}\right)=l+k \Rightarrow f\left(v_{2}\right)=N_{f_{m}}\left(v_{2}\right)=l+k-m$ $(\because l+k>m)$.

- $N_{f}\left(v_{3}\right)=f\left(v_{2}\right)+f\left(v_{4}\right)=l+k-m+f\left(v_{4}\right) \Rightarrow k=f\left(v_{3}\right)=N_{f_{m}}\left(v_{3}\right) \equiv$ $l+k-m+f\left(v_{4}\right)(\bmod m) \Rightarrow f\left(v_{4}\right)=m-l(\because m>m-l>0)$.

- $N_{f}\left(v_{4}\right)=f\left(v_{3}\right)+f\left(v_{5}\right)=k+f\left(v_{5}\right)$. $\Rightarrow m-l=f\left(v_{4}\right)=N_{f_{m}}\left(v_{4}\right) \equiv$ $k+f\left(v_{5}\right)(\bmod m) \Rightarrow f\left(v_{5}\right)=2 m-l-k .(\because m>2 m-(l+k)>0)$.

- $N_{f}\left(v_{5}\right)=f\left(v_{4}\right)+f\left(v_{6}\right)=m-l+f\left(v_{6}\right)$. $\Rightarrow 2 m-l-k=f\left(v_{5}\right)=$ $N_{f_{m}}\left(v_{5}\right) \equiv m-l+f\left(v_{6}\right)(\bmod m) \Rightarrow f\left(v_{6}\right)=m-k$.

- $N_{f}\left(v_{6}\right)=f\left(v_{5}\right)+f\left(v_{7}\right)=2 m-l-k+f\left(v_{7}\right)$. $\Rightarrow m-k=f\left(v_{6}\right)=$ $N_{f_{m}}\left(v_{6}\right) \equiv 2 m-l-k+f\left(v_{7}\right)(\bmod m) \Rightarrow f\left(v_{7}\right)=l$.

Thus in either of the cases, $f\left(v_{7}\right)=l=f\left(v_{1}\right)$ and hence $v_{7}=v_{1}$ (since $f$ is injective), a contradiction.

We now consider the small cases where $n \leq 5$. When $n=3$, it is shown in [10] that the cycle $C_{3}$ is not an outer mod sum graph and hence, by Lemma 2.1 and Lemma 2.2, $C_{3}$ is not a SSOMSG and hence not a SOMSG. When $n=4$, it follows by Theorem 2.5 that the graph $C_{4}$ is not a SSOSMG. Finally, when $n=5$, we see by labeling $f\left(v_{1}\right)=l$ and $f\left(v_{3}\right)=k$ as in the above two cases respectively that $m-l-k=f\left(v_{5}\right)=f\left(v_{1}\right)+f\left(v_{4}\right)=l+m-l(\bmod m)$ 
or $2 m-l-k=f\left(v_{5}\right)=f\left(v_{1}\right)+f\left(v_{4}\right)=l+m-l(\bmod m)$. In either of these cases, we get $l \equiv k(\bmod m) \Rightarrow l=k($ as $l, k<m)$, which is a contradiction to the fact that $f$ is injective.

Conjecture 4.2. For every integer $n \geq 3(n \neq 6)$, the cycle $C_{n}$ is not a SSOMSG.

\section{SOMSL and SSOMSL of a Complete graph}

Theorem 5.1. For every integer $n \geq 2(n \neq 3)$, the complete graph $K_{n}$ is a SSOMSG under addition modulo $m$, where $m=n+1$ if $n$ is even, and $m=\frac{(n+1)(n-2)}{2}$ if $n$ is odd.

Proof. When $n=3, K_{3} \equiv C_{3}$, is not an outer mod sum graph. Consider a complete graph $K_{n}$ on $n \neq 3$ vertices $v_{1}, v_{2}, \ldots, v_{n}$. Define $f: V\left(K_{n}\right) \rightarrow$ $\{1,2, \ldots, n\}$ as $f\left(v_{i}\right)=i$ for all $i, 1 \leq i \leq n$. It is shown in [10] that the function $f$ so defined is an outer mod sum labeling under addition modulo $n+1$ if $n$ is even and under addition modulo $\left(\frac{(n+1)(n-2)}{2}\right)$ if $n$ is odd. Therefore each vertex $v_{i}$ has a working vertex. Further, for all $i, 1 \leq i \leq n, N_{f}\left(v_{i}\right)=$ $\sum_{k=1, k \neq i}^{n} f\left(v_{k}\right)=\sum_{k=1}^{n} f\left(v_{k}\right)-f\left(v_{i}\right)=\sum_{k=1}^{n} k-i=\frac{n(n+1)}{2}-i$. Now when $n$ is even, $N_{f}\left(v_{i}\right)=\frac{n(n+1)}{2}-i \equiv(-i)(\bmod n+1) \Rightarrow N_{f}\left(v_{i}\right) \equiv n+1-i(\bmod n+1) \Rightarrow$ $N_{f_{n+1}}\left(v_{i}\right)=n+1-i=f\left(v_{n+1-i}\right)$ or equivalently, $N_{f_{n+1}}\left(v_{n+1-i}\right)=f\left(v_{i}\right)$. When $n$ is odd, $N_{f}\left(v_{i}\right)=\frac{n(n+1)}{2}-i=\frac{(n-2+2)(n+1)}{2}-i=\frac{(n-2)(n+1)}{2}+n+1-i \equiv n+1-i$ $\left(\bmod \frac{(n-2)(n+1)}{2}\right) \Rightarrow N_{\frac{(n+1)(n-2)}{2}}\left(v_{i}\right)=n+1-i=f\left(v_{n+1-i}\right)$ or equivalently,

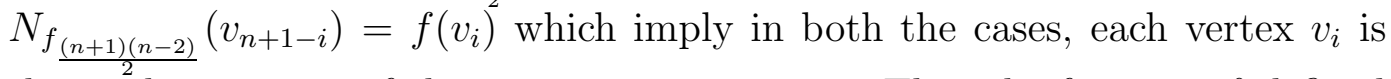
the working vertex of the unique vertex $v_{n+1-i}$. Thus the function $f$ defined above is a semi strong outer mod sum labeling under addition modulo $n+1$ if $n$ is even and under addition modulo $\left(\frac{(n+1)(n-2)}{2}\right)$ when $n$ is odd. Hence the theorem.

Theorem 5.2. For every integer $n \geq 3$, the complete graph $K_{n}$ is not a strong outer mod sum graph.

Proof. In view of Theorem 3.5 and Theorem 4.1, it suffices to prove the result for $n>3$. If possible, suppose $K_{n}(n>3)$ is a SOMSG and $f$ be a SOMSL of $K_{n}$ under modulo $m$. Then, by Theorem 2.6, it follows that $(n-2) \sum_{k=1}^{n} f\left(v_{i}\right) \equiv 0$ $(\bmod m)$. Now, we have the following cases.

Case 1: $\operatorname{gcd}(n-2, m)=1$

In this case $\sum_{k=1}^{n} f\left(v_{i}\right) \equiv 0(\bmod m) \Rightarrow f\left(v_{i}\right) \equiv-\left(f\left(v_{1}\right)+f\left(v_{2}\right)+\ldots+\right.$ $\left.f\left(v_{i-1}\right)+f\left(v_{i+1}\right) \ldots+f\left(v_{n}\right)\right)(\bmod m) \Rightarrow f\left(v_{i}\right) \equiv-\left(f\left(v_{i}\right)\right)(\bmod m) \Rightarrow$ $2 f\left(v_{i}\right) \equiv 0(\bmod m) \Rightarrow f\left(v_{i}\right) \equiv 0\left(\bmod \frac{m}{2}\right)\left(\because 0<f\left(v_{i}\right)<m\right.$ and 
hence $2 \mid m)$, for every $i, 1 \leq i \leq n \Rightarrow f\left(v_{i}\right)=\frac{m}{2}$ for each $i$, which is a contradiction (since $f$ is injective).

Case 2: $\operatorname{gcd}(n-2, m) \neq 1$

Let $\operatorname{gcd}(n-2, m)=k>1$. Then $\operatorname{gcd}\left(\frac{n-2}{k}, \frac{m}{k}\right)=1$, so $\sum_{k=1}^{n} f\left(v_{i}\right) \equiv$ $0\left(\bmod \frac{m}{k}\right) \Rightarrow f\left(v_{i}\right) \equiv-\left(f\left(v_{1}\right)+f\left(v_{2}\right)+\ldots+f\left(v_{i-1}\right)+f\left(v_{i+1}\right) \ldots+\right.$ $\left.f\left(v_{n}\right)\right)\left(\bmod \frac{m}{k}\right) \Rightarrow f\left(v_{i}\right) \equiv-\left(f\left(v_{i}\right)\right)\left(\bmod \frac{m}{k}\right) \Rightarrow 2 f\left(v_{i}\right) \equiv 0\left(\bmod \frac{m}{k}\right)$. Now if $\left(\frac{m}{k}, 2\right)=1$, then $f\left(v_{i}\right) \equiv 0\left(\bmod \frac{m}{k}\right)$ for every $i, 1 \leq i \leq n$, which is a contradiction, otherwise $\left(\frac{m}{k}, 2\right)=2 \Rightarrow\left(\frac{m}{2 k}, 1\right)=1$, then $f\left(v_{i}\right) \equiv 0(\bmod$ $\frac{m}{2 k}$ ) for every $i, 1 \leq i \leq n$, which is again a contradiction (since in either of the cases $1<k \leq n-2<m$ and $f$ is injective).

Hence the theorem.

ACKNOWLEDGEMENTS. Authors are very much thankful to the Management and the Principal of Dr. Ambedkar Institute of Technology, Bangalore for their constant support and encouragement during the preparation of this paper.

\section{References}

[1] J. Boland, R. Laskar, C. Turner, and G. Domke, On mod sum graphs, Congr. Numer., 70 (1990) 131-135.

[2] F. Buckley and Frank Harary, Distance in Graphs, AddisonWesley,(1990).

[3] Gary Chartrand and Ping Zhang, Introduction to Graph theory, Tata McGraw-Hell Edition (2006).

[4] Z. Chen, Harary's conjectures on integral sum graphs, Disc. Math., 160(1996) 241-244.

[5] Wenqing Dou and Jingzhen Gao, The (mod, integral) sum number of fans and $K_{n, n}-E\left(n K_{2}\right)$, Discrete Mathematics, 306 (2006) 2655-2669.

[6] Joseph A. Gallian, A dynamic survey of graph labeling, The Electronic Journal of Combinatorics, \# DS6,(2009),1-219.

[7] Hartsfield Gerhard and Ringel, Pearls in Graph Theory, Academic Press, USA, 1994.

[8] Frank Harary, Sum graph and difference graphs, Congr. Numer., 72 (1990) 101-108. 
[9] Frank Harary, Sum graph over all the integers,Disc. Math.,124(1994) 99105.

[10] Jayalakshmi M, B. Sooryanarayana, P Devadas Rao, Outer Mod Sum Labelings of a Graph, Internatial Journal of Information Science and Computer Mathematics, Volume 2, Number 2, 2010, pp87-102.

[11] K. N. Meera and B. Sooryanarayana, Optimal Outer Sum number of a Graphs,International Journal of Combinatorial graph theory and applications, Vol 4, No. 1, (Jan-june2011)pp 23-35.

[12] A. V. Pyatkin, New formula for the sum number for the complete bipartite graphs, Disc. Math., 239 (2001) 155-160.

[13] Joe Ryan, Exclusive sum labeling of graphs: A survey, AKEC International Journal of Graphs.Combinatorics.,Vol 6, No. 1, (2009) 113-126.

[14] B. Sooryanarayana, Manjula K and Vishu Kumar M, Outer Sum Labeling of a Graph, International Journal of Combinatorial graph theory and applications .,Vol 4, No. 1, (2011)37-60.

[15] Martin Sutton and Anna Draganova and Mirka Miller, Mod Sum Number of Wheels, Ars Combinatoria, 63(2002) 273-287.

[16] Haiying Wang, The sum numbers and integral sum number of the graph $K_{1, n} \backslash E\left(K_{1, n}\right)$, Disc. Math. 309(2009) 4137-4143.

Received: August, 2012 